\title{
Measurement of retinal nerve fiber layer thickness in eyes with optic disc swelling by using scanning laser polarimetry and optical coherence tomography
}

\author{
This article was published in the following Dove Press journal: \\ Clinical Ophthalmology \\ 19 December 2013 \\ Number of times this article has been viewed
}

\section{Masayuki Hata \\ Kazuaki Miyamoto \\ Akio Oishi \\ Yugo Kimura \\ Satoko Nakagawa \\ Takahiro Horii \\ Nagahisa Yoshimura}

Department of Ophthalmology and Visual Sciences, Kyoto University Graduate School of Medicine, Kyoto, Japan
Correspondence: Masayuki Hata Department of Ophthalmology and Visual Sciences, Kyoto University Graduate School of Medicine, Sakyo-ku,

Kyoto 606-8507, Japan

Tel +81757513250

Fax +81757520933

Email trj74h6@kuhp.kyoto-u.ac.jp
Background: The retinal nerve fiber layer thickness (RNFLT) in patients with optic disc swelling of different etiologies was compared using scanning laser polarimetry (SLP) and spectral-domain optical coherence tomography (OCT).

Methods: Forty-seven patients with optic disc swelling participated in the cross-sectional study. Both GDx SLP (enhanced corneal compensation) and Spectralis spectral-domain OCT measurements of RNFLT were made in 19 eyes with papilledema (PE), ten eyes with optic neuritis $(\mathrm{ON})$, and 18 eyes with nonarteritic anterior ischemic optic neuropathy (NAION) at the neuro-ophthalmology clinic at Kyoto University Hospital. Differences in SLP (SLP-RNFLT) and OCT (OCT-RNFLT) measurements among different etiologies were investigated.

Results: No statistical differences in average OCT-RNFLT among PE, ON, and NAION patients were noted. Average SLP-RNFLT in NAION patients was smaller than in PE $(P<0.01)$ or ON $(P=0.02)$ patients. When RNFLT in each retinal quadrant was compared, no difference among etiologies was noted on OCT, but on SLP, the superior quadrant was thinner in NAION than in $\mathrm{PE}(P<0.001)$ or $\mathrm{ON}(P=0.001)$ patients. Compared with age-adjusted normative data of SLPRNFLT, average SLP-RNFLT in PE $(P<0.01)$ and ON $(P<0.01)$ patients was greater. Superior SLP-RNFLT in NAION patients was smaller $(P=0.026)$. The ratio of average SLP-RNFLT to average OCT-RNFLT was smaller in NAION than in PE $(P=0.001)$ patients.

Conclusion: In the setting of RNFL thickening, despite increased light retardance in PE and ON eyes, SLP revealed that NAION eyes have less retardance, possibly associated with ischemic axonal loss.

Keywords: optic disc swelling, scanning laser polarimetry, optical coherence tomography

\section{Introduction}

The retinal nerve fiber layer (RNFL) is composed of retinal ganglion cell (RGC) axons. The circumpapillary RNFL (cpRNFL), in particular, comprises all nerve fibers entering the optic nerve disc. Therefore, evaluating the cpRNFL is useful in detecting RGC damage and in understanding the pathophysiology of neuro-ophthalmic disease. ${ }^{1,2}$ Thickness of the cpRNFL is a useful measure, and recent advances in diagnostic equipment allow for noninvasive measurement of RNFL thickness (RNFLT). ${ }^{3,4}$

Different types of instruments are available for measuring cpRNFLT. Optical coherence tomography (OCT), a near-infrared light-based imaging technique, allows for microscopic cross-sectional imaging of biological systems. ${ }^{5}$ An optical signal transmitted through, or reflected from, the human retina contains time-to-flight information, which 
in turn yields spatial information about tissue microstructure. Compared to conventional time-domain OCT (TD-OCT), the recent advances in spectral-domain OCT (SD-OCT) enable faster acquisition speed and fewer motion artifacts in the images, which provides reproducible RNFLT measurements. ${ }^{6,7}$

Scanning laser polarimetry (SLP) measures the change in light polarization (retardation) as light passes through a medium, in this case, the cornea and the RNFL. Weinreb et $\mathrm{al}^{8}$ reported a correlation between SLP values and histopathologic measurements in nonhuman retinas. In RNFL, axonal cell membranes, microtubules, neurofilaments, and mitochondria may contribute to tissue birefringence. ${ }^{9-11}$ Therefore, SLP measurements reflect the birefringent properties of the RNFL and are not a substitute for actual RNFLT measurements. ${ }^{12}$

These two instruments have the possibility of evaluating different elements in measuring RNFLT. The OCT measurements reflect the anatomic thickness of the cpRNFL and include both intracellular and extracellular components. ${ }^{13}$ In contrast, SLP measurements provide information on intraaxonal microtubule and neurofilament structure. ${ }^{9-11}$

Both OCT and SLP measurements show similar tendency of RNFL thinning in glaucoma and other diseases that involve optic nerve atrophy. There is a strong correlation between the two measures, ${ }^{14}$ which means that a reduction in tissue birefringence accompanies RNFL thinning. However, the correlation between the two measurements in the setting of disc swelling (eg, papilledema $[\mathrm{PE}]$, anterior ischemic optic neuropathy [AION], or optic neuritis [ON]) had remained unknown.

Recently, Kupersmith et $a{ }^{15}$ reported a disparity between SLP and OCT measurements in eyes with nonarteritic AION (NAION). Interestingly, SLP measurements of RNFLT revealed an RNFL thinning in eyes with NAION, in contrast to an increase in RNFLT measured mainly by TD-OCT in eyes with NAION or other conditions of optic disc swelling, while PE and ON cases showed slight increase in SLPRNFLT. However, it has also been reported that RNFLT measurements differ between TD-OCT and SD-OCT, and that these measurements are not interchangeable. ${ }^{7,16}$ Therefore, in the present study, we used SD-OCT, which provides both faster scan acquisition and higher scan resolution than TD-OCT, to investigate differences in cpRNFLT measurements obtained by SD-OCT (OCT-RNFLT) and SLP (SLP-RNFLT) in the setting of optic disc swelling.

\section{Materials and methods}

\section{Patients}

Ethics approval was granted by the Institutional Review Board of Kyoto University Graduate School of Medicine.
We retrospectively reviewed the clinical records of 47 patients with optic disc swelling caused by PE, NAION, or ON, who presented at the neuro-ophthalmology clinic at Kyoto University Hospital between April 2009 and March 2012. To be included in analyses, both ON and NAION had to be of new onset (vision loss or visual field defect within 2 weeks), and PE cases had to have no visual dysfunction or have it limited to blind spot enlargement. Eyes with other ocular diseases, except mild cataract, were excluded.

\section{Diagnostic determinations and classifications of etiologies}

All patients underwent various eye examinations to determine the cause of optic disc swelling. At the first visit, evaluations included visual acuity, slit lamp examination, fundus photography, perimetry, Spectralis SD-OCT (Heidelberg Spectralis OCT; Heidelberg Engineering Inc, Heidelberg, Germany), and SLP (GDx Nerve Fiber Analyzer; Carl Zeiss Meditec AG, Jena, Germany). An experienced neuro-ophthalmologist made all diagnoses (KM). A diagnosis of NAION was based on clinical signs and symptoms alone, which included acute/ subacute monocular visual impairment at an age of $\geq 45$ years, absence of pain, and absence of giant cell arteritis or multiple sclerosis. A diagnosis of $\mathrm{ON}$ was also based on clinical grounds alone, and signs and symptoms included acute/subacute monocular visual impairment occurring between 15-45 years of age, retrobulbar pain and/or pain with eye movement, and a history of multiple sclerosis. All patients with $\mathrm{ON}$ underwent magnetic resonance imaging with gadolinium enhancement. A diagnosis of PE was made if patients had intracranial hypertension, as confirmed by lumbar puncture or magnetic resonance imaging with gadolinium enhancement.

\section{Scanning laser polarimetry and optical coherence tomography}

All SLP-RNFLT measurements were made in nondilated pupils on an SLP system equipped with enhanced corneal compensation research software (GDx ECC; Carl Zeiss Meditec AG). Five SLP parameters, which provided absolute measurements of RNFL retardation, were analyzed. These included average RNFLT measurements of the entire circumpapillary region (temporal-superior-nasal-inferior-temporal [TSNIT], average SLP-RNFLT), the superior quadrant (superior SLP-RNFLT), the inferior quadrant (inferior SLPRNFLT), the temporal quadrant (temporal SLP-RNFLT), and the nasal quadrant (nasal SLP-RNFLT). The ellipse average represents average thickness of the retinal nerve fiber in a 
10-pixel-wide band with a diameter 1.75 times that of the optic nerve.

Spectralis SD-OCT was used in high-resolution mode to measure RNFL thickness with the built-in RNFL/optic disc protocol. Five OCT parameters were used to assess RNFLT and included average cpRNFL (average OCTRNFLT), average superior (superior OCT-RNFLT), average inferior (inferior OCT-RNFLT), average temporal (temporal OCT-RNFLT), and average nasal (nasal OCT-RNFLT) measurements. These parameters were automatically generated by both the SLP and SD-OCT software.

As for SD-OCT, at least two scans were performed by the same experienced operator after the patient's pupils had been dilated. To enhance the SD-OCT signal-to-noise ratio, over 40 scans were averaged to obtain the final recording. Only images that were well-centered on the optic disc and had good quality were chosen to be included in analyses. A single physician $(\mathrm{MH})$, who was masked to the patient's clinical condition, made the decision to include or exclude patient scans. For SLP scans, only images with a quality score $\geq 7$ were used, and results were compared with normative data from the GDx database. All SLP-RNFLT measurements were adjusted for age by using the best-fit line obtained from 540 normal eyes. ${ }^{17}$ The following equations were used:

$$
\begin{gathered}
\text { TSNIT average: } \mathrm{y}=-0.053 \times \text { age }+58.563 \\
\text { Superior average: } \mathrm{y}=-0.1164 \times \text { age }+73.444 \\
\text { Inferior average: } \mathrm{y}=-0.1047 \times \text { age }+70.356
\end{gathered}
$$

\section{Data analyses}

Data are presented as mean \pm standard deviation. Statistical analyses were performed using statistical software (SPSS Statistics Desktop, version 19.0.0; IBM Corporation, Armonk, NY, USA). Patient means between disease groups were compared using unpaired Student's $t$-tests and one-way ANOVA with Bonferroni correction. Bivariate relationships were examined using Spearman correlation coefficients. Statistical significance was defined as $P \leq 0.05$.

\section{Results}

Nineteen eyes (ten patients) with PE, 18 eyes (17 patients) with NAION, and ten eyes (eight patients) with ON were included in analyses. Mean subject age at disease onset was 41.8 \pm 17.7 years in PE patients, $61.8 \pm 12.1$ years in NAION patients, and $34.5 \pm 12.3$ years in $\mathrm{ON}$ patients.

Average, superior, inferior, temporal, and nasal SLPRNFLT and OCT-RNFLT in eyes with each disease are shown in Table 1. Average SLP-RNFLT and OCT-RNFLT were significantly correlated in eyes with PE $(r=0.55$, $P=0.015)$ (Figure 1) and NAION $(r=0.68, P=0.006)$ (Figure 1), but not in eyes with $\mathrm{ON}(P=0.17)$.

As for visual fields, all subjects with $\mathrm{PE}$ had normal visual fields or only blind spot enlargement, consistent with the inclusion/exclusion criteria. In eyes with $\mathrm{ON}$, visual field testing results showed a central scotoma in five cases and general visual field defects in three eyes. Nine of 18 eyes with NAION had an inferior altitudinal field defect, two eyes had a superior altitudinal field defect, two eyes had a central scotoma,

\begin{tabular}{|c|c|c|c|c|c|c|}
\hline & NAION $(n=\mid 8)$ & PE $(n=19)$ & ON $(n=10)$ & $\boldsymbol{P}$-value* & $\boldsymbol{P}$-value ${ }^{\dagger}$ & $P$-value ${ }^{\ddagger}$ \\
\hline \multicolumn{7}{|l|}{ Average } \\
\hline SLP & $51.7 \pm 10.9$ & $63.5 \pm 10.5$ & $62.7 \pm 5.3$ & 0.002 & 0.020 & 1.000 \\
\hline SD-OCT & $162.5 \pm 55.4$ & $169.8 \pm 47.0$ & $145.6 \pm 24.7$ & 1.000 & 1.000 & 0.621 \\
\hline \multicolumn{7}{|l|}{ Superior } \\
\hline SLP & $58.5 \pm 13.6$ & $73.5 \pm 8.6$ & $76.0 \pm 10.5$ & $<0.001$ & 0.001 & 1.000 \\
\hline SD-OCT & $194.0 \pm 56.9$ & $208.8 \pm 66.4$ & $184.6 \pm 59.6$ & 1.000 & 1.000 & 1.000 \\
\hline \multicolumn{7}{|l|}{ Inferior } \\
\hline SLP & $66.6 \pm 17.0$ & $75.1 \pm 16.8$ & $78.2 \pm 11.2$ & 0.337 & 0.209 & 1.000 \\
\hline SD-OCT & $210.7 \pm 60.6$ & $235.5 \pm 88.4$ & $200.4 \pm 33.1$ & 0.949 & 1.000 & 0.686 \\
\hline \multicolumn{7}{|l|}{ Temporal } \\
\hline SLP & $28.2 \pm 12.2$ & $38.9 \pm 15.2$ & $33.1 \pm 13.0$ & 0.064 & 1.000 & 0.863 \\
\hline SD-OCT & $132.5 \pm 58.2$ & I $4.9 \pm 38.1$ & $89.8 \pm 17.1$ & 0.753 & 0.076 & 0.482 \\
\hline \multicolumn{7}{|l|}{ Nasal } \\
\hline SLP & $31.5 \pm 10.9$ & $44.2 \pm 17.0$ & $36.1 \pm 9.1$ & 0.020 & 1.000 & 0.403 \\
\hline SD-OCT & $112.5 \pm 76.6$ & $119.8 \pm 62.2$ & $106.1 \pm 32.4$ & 1.00 & 1.00 & 1.00 \\
\hline
\end{tabular}

Table I Retinal nerve fiber layer thickness as measured by scanning laser polarimetry and spectral-domain optical coherence tomography

Notes: *One-way ANOVA with Bonferroni correction between NAION and PE; †one-way ANOVA with Bonferroni correction between NAION and ON; ${ }^{\ddagger}$ one-way ANOVA with Bonferroni correction between PE and ON.

Abbreviations: ANOVA, analysis of variance; NAION, nonarteritic anterior ischemic optic neuropathy; ON, optic neuritis; PE, papilledema; SD-OCT, spectral-domain optical coherence tomography; SLP, scanning laser polarimetry. 


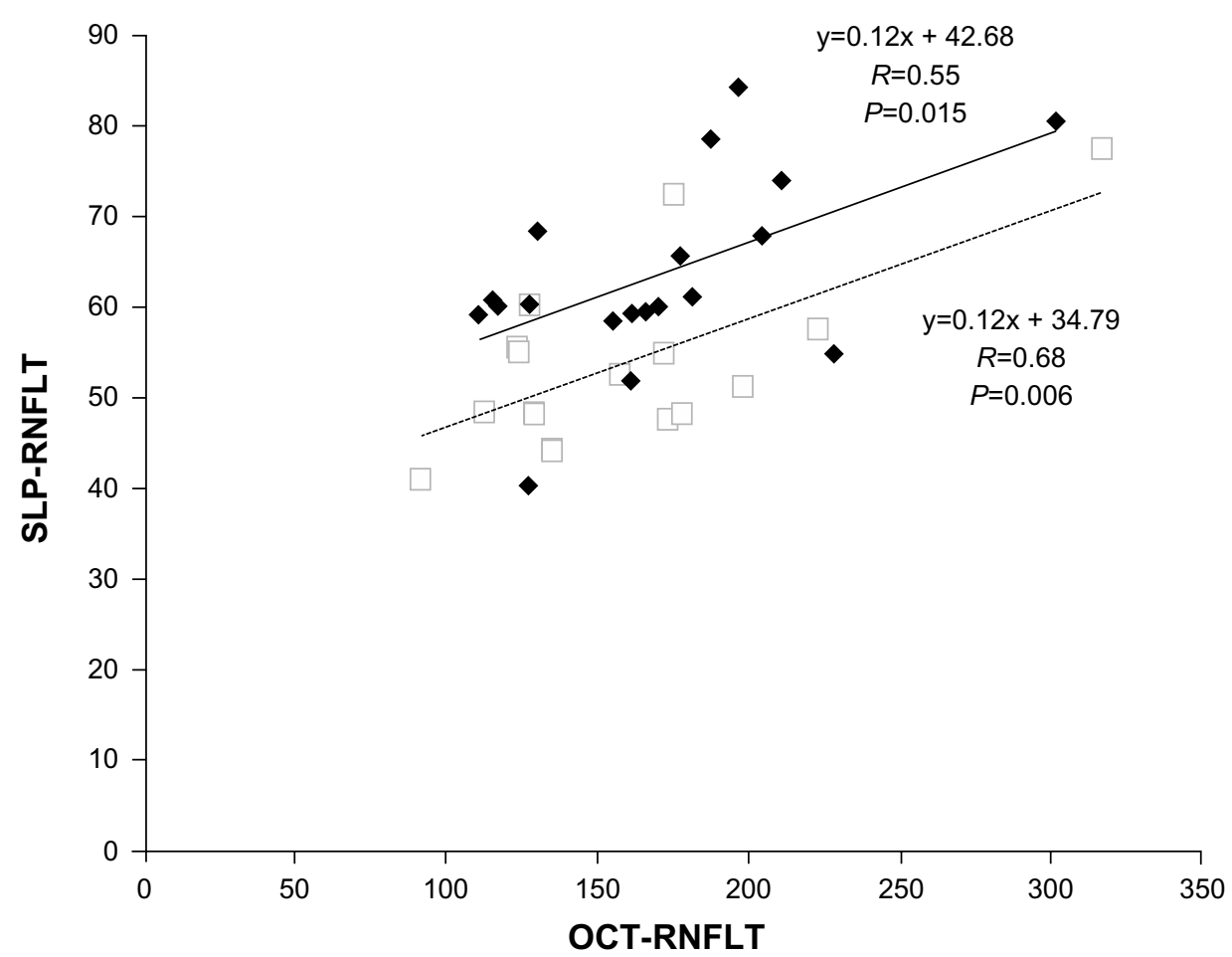

Figure I Scatter plot of average retinal nerve fiber layer thickness (RNFLT) measurements obtained by Spectralis SD-OCT and SLP with GDx ECC in eyes with papilledema $(\mathrm{PE}, \bullet)$ and nonarteritic anterior ischemic optic neuropathy (NAION, $\square$ ).

Notes: The best-fit lines for eyes with PE (-) and eyes with NAION (-) are shown. Data for eyes with optic neuritis is not shown because the correlation between OCT and SLP measurements of RNFLT was not significant. Spectralis SD-OCT, Heidelberg Engineering Inc, Heidelberg, Germany; GDx ECC, Carl Zeiss Meditec AG, Jena, Germany.

Abbreviations: OCT, optical coherence tomography; SD-OCT, spectral-domain optical coherence tomography; SLP, scanning laser polarimetry.

and three eyes had a general visual defect. Representative cases with ON and NAION are shown in Figures 2 and 3.

Eyes with NAION had a significantly smaller average SLP-RNFLT than eyes with PE $(P=0.002)$ or ON $(P=0.02)$ (Table 1). Divided into four quadrants of SLP-RNFLT, there was no difference in inferior and temporal quadrants between NAION and other etiologies. However, the superior quadrant was significantly thinner in eyes with NAION, compared with eyes that had PE $(P<0.001)$ or ON $(P=0.001)$. In the nasal quadrant, SLP-RNFLT was significantly smaller in eyes with NAION than in eyes with PE $(P=0.020)$. There were no significant differences in OCT-RNFLT results among the three diagnosis groups in any of the four quadrants.

Age-adjusted SLP-RNFLT in normal eyes was obtained from each linear regression line of normative data. When adjusted for age of PE patients (mean age $=41.8$ years), average SLP-RNFLT was 56.3, superior SLP-RNFLT was 68.6, and inferior SLP-RNFLT was 66.0. When adjusted for age of ON patients (mean age $=34.5$ years), average SLPRNFLT was 56.7, superior SLP-RNFLT was 69.4, and inferior SLP-RNFLT was 66.7. When adjusted for age of NAION patients (mean age $=61.8$ years), average SLP-RNFLT was 55.3, superior SLP-RNFLT was 66.3, and inferior SLP-RNFLT was 63.9. Average SLP-RNFLT was significantly greater in eyes with $\mathrm{ON}(P=0.006)$ or PE $(P=0.008)$ than in age-matched normal eyes. This was not the case for eyes with NAION ( $P=0.182)$ (Table 2). Divided into superior and inferior quadrants, both the superior $(P=0.021)$ and inferior $(P=0.03)$ quadrants had a greater SLP-RNFLT in eyes with $\mathrm{PE}$ than in normal eyes. In eyes with $\mathrm{ON}$, the inferior quadrant had a SLP-RNFLT significantly greater than normal $(P=0.01)$. In eyes with NAION, however, superior quadrant SLP-RNFLT was significantly smaller than normal $(P=0.03)$.

Data from eyes with NAION were subdivided into those that had an inferior altitudinal field defect (nine eyes) and those that had other types of visual field defects (nine eyes). Average SLP-RNFLT was not significantly different between the two groups ( $49.7 \pm 6.8$ versus $53.8 \pm 14.0, P=0.438$ ), but superior SLP-RNFLT was significantly smaller in eyes with an inferior altitudinal field defect $(51.0 \pm 11.7$ versus $65.9 \pm 11.5, P=0.015$ ).

Although RNFLT in eyes with PE and NAION were similar when measured with OCT, measurements made with SLP showed significant differences between eyes with PE 

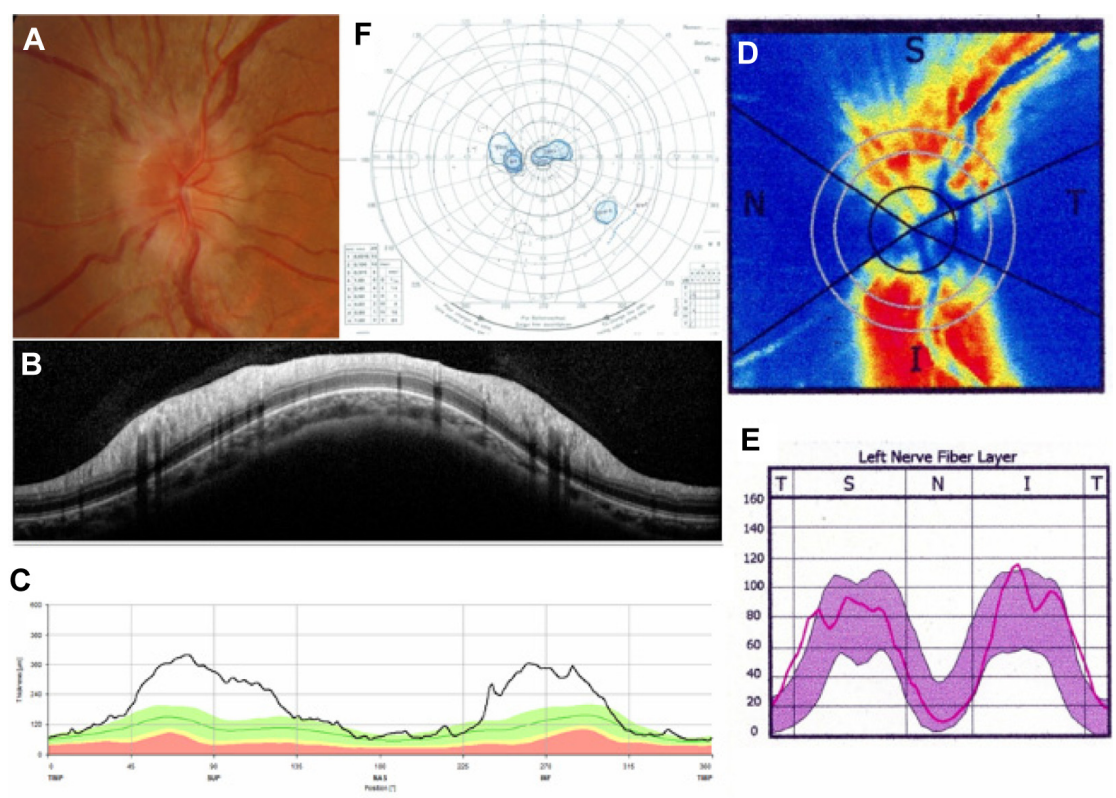

Figure 2 Representative case of optic neuritis.

Notes: A fundus photograph (A) shows optic disc swelling in the left eye. Spectralis SD-OCT (B and C) and SLP with GDx ECC (D and E) reports demonstrate RNFL thickening. Goldmann perimetry (F) shows a central scotoma. Spectralis SD-OCT, Heidelberg Engineering Inc, Heidelberg, Germany; GDx ECC, Carl Zeiss Meditec AG, Jena, Germany. Abbreviations: I, inferior; N, nasal; RNFL, retinal nerve fiber layer thickness; S, superior; SD-OCT, spectral-domain optical coherence tomography; T, temporal; SLP, scanning laser polarimetry.

and eyes with NAION. To compare SLP-RNFLT measurements with OCT-RNFLT measurements, we calculated the ratio of average SLP-RNFLT to OCT-RNFLT. This ratio was significantly higher in eyes with PE (0.39 \pm 0.09$)$, compared to eyes with NAION $(0.28 \pm 0.08, P=0.001)$.

\section{Discussion}

In the present study, we report a disparity in SD-OCT and SLP RNFLT measurements in eyes with NAION, even in the acute stage of the disease. There was no difference in average OCT-RNFLT values among ON, NAION, and PE, but SLP
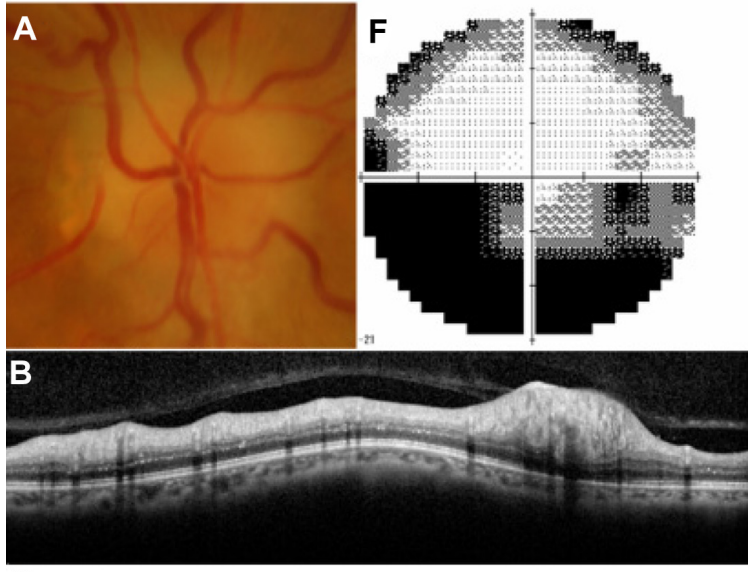

C

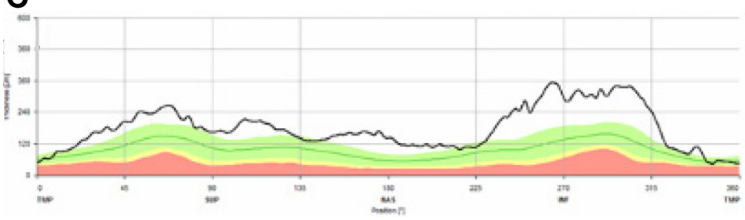

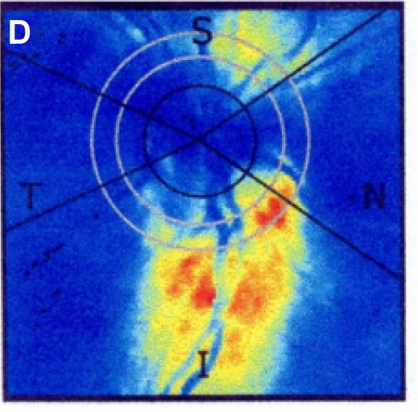

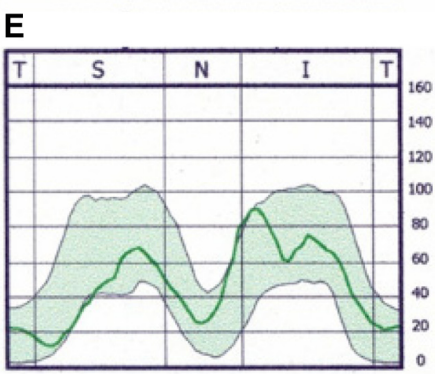

Figure 3 Representative case of nonarteritic anterior ischemic optic neuropathy.

Notes: Fundus photograph (A) shows optic disc swelling with disc hemorrhage in the right eye. Spectralis SD-OCT images (B and C) show RNFL thickening in the superior quadrant, while SLP with GDx ECC imaging (D and E) shows slight RNFL thinning in the superior quadrant. Visual field testing (F) shows an inferior altitudinal field defect. Spectralis SD-OCT, Heidelberg Engineering Inc, Heidelberg, Germany; GDx ECC, Carl Zeiss Meditec AG, Jena, Germany.

Abbreviations: I, inferior; N, nasal; RNFL, retinal nerve fiber layer; S, superior; SD-OCT, spectral-domain optical coherence tomography; T, temporal; SLP, scanning laser polarimetry. 
Table 2 Comparison of retinal nerve fiber layer thickness, as measured with scanning laser polarimetry, between eyes with optic disc swelling and age-adjusted normative data

\begin{tabular}{|c|c|c|c|}
\hline & $\begin{array}{l}\text { NAION versus } \\
\text { normal }\end{array}$ & $\begin{array}{l}\text { PE versus } \\
\text { normal }\end{array}$ & $\begin{array}{l}\text { ON versus } \\
\text { normal }\end{array}$ \\
\hline \multicolumn{4}{|l|}{ Average } \\
\hline SLP & $\begin{array}{l}51.7 \pm 10.9 \\
\text { versus } 55.3\end{array}$ & $\begin{array}{l}63.5 \pm 10.5 \\
\text { versus } 56.3\end{array}$ & $\begin{array}{l}62.7 \pm 5.3 \\
\text { versus } 56.7\end{array}$ \\
\hline$P$-value* & 0.182 & 0.008 & 0.006 \\
\hline \multicolumn{4}{|l|}{ Superior } \\
\hline SLP & $\begin{array}{l}58.5 \pm 13.6 \\
\text { versus } 66.3\end{array}$ & $\begin{array}{l}73.5 \pm 8.6 \\
\text { versus } 68.6\end{array}$ & $\begin{array}{l}76.0 \pm 10.5 \\
\text { versus } 69.4\end{array}$ \\
\hline$P$-value* & 0.026 & 0.021 & 0.079 \\
\hline \multicolumn{4}{|l|}{ Inferior } \\
\hline SLP & $\begin{array}{l}66.6 \pm 17.0 \\
\text { versus } 63.9\end{array}$ & $\begin{array}{l}75.1 \pm 16.8 \\
\text { versus } 66.0\end{array}$ & $\begin{array}{l}78.2 \pm 11.2 \\
\text { versus } 66.7\end{array}$ \\
\hline$P$-value* & 0.508 & 0.030 & 0.010 \\
\hline
\end{tabular}

Note: *Student's t-test.

Abbreviations: NAION, nonarteritic anterior ischemic optic neuropathy; ON, optic neuritis; PE, papilledema; SLP, scanning laser polarimetry.

measurements showed that average RNFLT in eyes with NAION was smaller than in eyes with ON or PE. Moreover, superior SLP-RNFLT in eyes with NAION was smaller than in normal eyes, which corresponded to functional changes, as evidenced by inferior visual field defects.

Spectral domain OCT, which evaluates anatomical RNFLT, reflects both intracellular and extracellular edema. Consistent with slit-lamp exam findings, both NAION and PE had a tendency of showing OCT-RNFL thickening, as compared with ON. On the other hand, SLP cannot provide exact RNFLT when optic disc swelling occurs, especially in eyes with NAION. SLP measures double pass phase retardation that is induced by birefringent structures, such as the microtubules in the RNFL, and converts the phase retardation to a thickness. In eyes with optic disc swelling, the RNFL does not lead to extra microtubules or extra birefringence, which in turn does not cause any extra double pass phase retardation. SLP is not sensitive to swelling of the nerve fiber, unless the axons are damaged and disappear, which should result in a lower signal. Therefore, SD-OCT is more suitable than SLP to quantify anatomical thickening in optic disc swelling. However, SD-OCT cannot distinguish between intracellular and extracellular edema, and it cannot detect axonal damage beneath the edema. Though NAION, $\mathrm{ON}$, and PE have distinct etiologies, we cannot differentiate among them with SD-OCT images alone.

SLP measures light retardation that is proportional to the number of microtubules. ${ }^{9-11}$ Axonal damage decreases the birefringence properties of RGCs. ${ }^{18}$ Colen et al ${ }^{19}$ reported axonal loss in a patient with acute NAION with the GDx, and it was followed by a gradual disappearance of nerve fiber tissue within several weeks. Eyes with NAION had thinner RNFL measurements on SLP than the other etiologies examined. This may indicate that early axonal damage is caused by the ischemia that occurs with NAION. SLP may aid us in differentiating underlying etiologies and help us to measure axonal damage in the setting of optic disc edema.

When compared with normative data, both $\mathrm{ON}$ and $\mathrm{PE}$ had slightly thicker RNFLs in SLP measurements. This increase in retardance was thought to be caused by increased organelle density and intra-axonal water content. ${ }^{20-22}$ Even in eyes with NAION, intracellular edema could influence retardance to some extent, and when compared to normative data, NAION showed no difference in average SLP-RNFLT. It is possible that axonal damage in NAION may be masked by intracellular edema in both SLP and SD-OCT measurements. We therefore examined the ratio of average SLP-RNFLT to OCT-RNFLT so as to examine the discrepancy between SLP measurements and anatomical thickness. As a result, even though OCT-RNFLT was quite similar in eyes with PE and NAION, there was a significant difference between these conditions on SLP measurements. The ratio of the two measurements may reveal the existence of axonal damage masked by intracellular edema, and comparing these results with those obtained in other conditions of similar anatomical edema may not only help in the diagnosis process, but add to our understanding of axonal loss in disease.

Kupersmith et a ${ }^{15}$ previously compared SLP-RNFLT and OCT-RNFLT measurements in optic disc swelling and demonstrated a discrepancy in eyes with NAION. Two types of OCT devices, TD-OCT and SD-OCT, were used in their study. The technological advances from TD-OCT to SDOCT have improved OCT scan speed. ${ }^{23,24}$ As a result, SDOCT can provide higher repeatability and reproducibility of RNFLT measurements than was possible with TD-OCT.,25 Differences in RNFL measurements between TD-OCT and SD-OCT are reported, and these measurements are not considered to be interchangeable. ${ }^{716}$ Therefore, we performed the present study with Spectralis SD-OCT and, in agreement with previous work, ${ }^{15}$ showed a difference between OCTRNFLT and SLP-RNFLT measurements.

There are some limitations to our study that should be acknowledged. In addition to the small number of patients studied, our study design was cross-sectional and retrospective. Prospective longitudinal studies in larger samples are needed to better understand how PE, ON, and NAION are represented on OCT and SLP. In addition, in patients with PE, disease onset was uncertain, and chronic cases may have been unintentionally 
included in our analyses. We tried to minimize this by including only those cases that had minimal visual impairment. On the flip side, this may have introduced a patient selection bias.

In conclusion, exact anatomical thickness in eyes with optic disc swelling could not be evaluated by SLP differently from OCT. In particular, eyes with early-stage NAION showed RNFL thinning on SLP measurements, while eyes with $\mathrm{ON}$ or PE did not. This may suggest that GDx SLP detects axonal damage, presumably caused by ischemia, even in the setting of optic disc swelling. The magnitude of the discrepancy between SLP and OCT measurements of RNFLT may be useful in differentiating diseases that are accompanied by optic disc swelling.

\section{Disclosure}

The authors report no conflicts of interest in this work.

\section{References}

1. Jonas JB, Dichtl A. Evaluation of the retinal nerve fiber layer. Surv Ophthalmol. 1996;40(5):369-378.

2. Pasol J. Neuro-ophthalmic disease and optical coherence tomography: glaucoma look-alikes. Curr Opin Ophthalmol. 2011;22(2):124-132.

3. Burk RO, Völcker HE. Current imaging of the optic disk and retinal nerve fiber layer. Curr Opin Ophthalmol. 1996;7(2):99-108.

4. Townsend KA, Wollstein G, Schuman JS. Imaging of the retinal nerve fibre layer for glaucoma. Br J Ophthalmol. 2009;93(2):139-143.

5. Huang D, Swanson EA, Lin CP, et al. Optical coherence tomography. Science. 1991;254(5035):1178-1181.

6. Wojtkowski M, Srinivasan V, Fujimoto JG, et al. Three-dimensional retinal imaging with high-speed ultrahigh-resolution optical coherence tomography. Ophthalmology. 2005;112(10):1734-1746.

7. Arthur SN, Smith SD, Wright MM, et al. Reproducibility and agreement in evaluating retinal nerve fibre layer thickness between Stratus and Spectralis OCT. Eye (Lond). 2011;25(2):192-200.

8. Weinreb RN, Dreher AW, Coleman A, Quigley H, Shaw B, Reiter K. Histopathologic validation of Fourier-ellipsometry measurements of retinal nerve fiber layer thickness. Arch Ophthalmol. 1990;108(4): $557-560$.

9. Huang XR, Knighton RW. Microtubules contribute to the birefringence of the retinal nerve fiber layer. Invest Ophthalmol Vis Sci. 2005;46(12): 4588-4593.

10. Zhou Q, Knighton RW. Light scattering and form birefringence of parallel cylindrical arrays that represent cellular organelles of the retinal nerve fiber layer. Appl Opt. 1997;36(10):2273-2285.
11. Knighton RW, Huang X, Zhou Q. Microtubule contribution to the reflectance of the retinal nerve fiber layer. Invest Ophthalmol Vis Sci. 1998;39(1):189-193.

12. Banks MC, Robe-Collignon NJ, Rizzo JF. Scanning laser polarimetry of edematous and atrophic optic nerve heads. Arch Ophthalmol. 2003;121(4):484-490.

13. Schuman JS, Pedut-Kloizman T, Pakter H, et al. Optical coherence tomography and histologic measurements of nerve fiber layer thickness in normal and glaucomatous monkey eyes. Invest Ophthalmol Vis Sci. 2007;48(8):3645-3654.

14. Medeiros FA, Zangwill LM, Bowd C, Weinreb RN. Comparison of the GDx VCC scanning laser polarimeter, HRT II confocal scanning laser ophthalmoscope, and stratus OCT optical coherence tomograph for the detection of glaucoma. Arch Ophthalmol. 2004;122(6):827-837.

15. Kupersmith MJ, Kardon R, Durbin M, Horne M, Shulman J. Scanning laser polarimetry reveals status of RNFL integrity in eyes with optic nerve head swelling by OCT. Invest Ophthalmol Vis Sci. 2012;53(4): 1962-1970.

16. Vizzeri G, Weinreb RN, Gonzalez-Garcia AO, et al. Agreement between spectral-domain and time-domain OCT for measuring RNFL thickness. Br J Ophthalmol. 2009;93(6):775-781.

17. Laser Diagnostic Technologies, Inc. RNFL Evaluation with the GDx VCC to Diagnose and Monitor Glaucoma. In: The GDx VCC Primer. San Diego, CA: Laser Diagnostic Technologies, Inc; 2004:20;17-25.

18. Weinreb RN, Shakiba S, Zangwill L. Scanning laser polarimetry to measure the nerve fiber layer of normal and glaucomatous eyes. Am J Ophthalmol. 1995;119(5):627-636.

19. Colen TP, van Everdingen JA, Lemij HG. Axonal loss in a patient with anterior ischemic optic neuropathy as measured with scanning laser polarimetry. Am J Ophthalmol. 2000;130(6):847-850.

20. Kupersmith MJ, Mandel G, Anderson S, Meltzer DE, Kardon R. Baseline, one and three month changes in the peripapillary retinal nerve fiber layer in acute optic neuritis: relation to baseline vision and MRI. J Neurol Sci. 2011;308(1-2):117-123.

21. Tso MO, Fine BS. Electron microscopic study of human papilledema. Am J Ophthalmol. 1976;82(3):424-434.

22. Hayreh SS. Pathogenesis of optic disc oedema in raised intracranial pressure. Trans Ophthalmol Soc UK. 1976;96(3):404-407.

23. van Velthoven ME, Faber DJ, Verbraak FD, van Leeuwen TG, de Smet MD. Recent developments in optical coherence tomography for imaging the retina. Prog Retin Eye Res. 2007;26(1):57-77.

24. Chen TC, Cense B, Pierce MC, et al. Spectral domain optical coherence tomography: ultra-high speed, ultra-high resolution ophthalmic imaging. Arch Ophthalmol. 2005;123(12):1715-1720.

25. Töteberg-Harms M, Sturm V, Knecht PB, Funk J, Menke MN Repeatability of nerve fiber layer thickness measurements in patients with glaucoma and without glaucoma using spectral-domain and time-domain OCT. Graefes Arch Clin Exp Ophthalmol. 2012;250(2): 279-287.
Clinical Ophthalmology

\section{Publish your work in this journal}

Clinical Ophthalmology is an international, peer-reviewed journal covering all subspecialties within ophthalmology. Key topics include: Optometry; Visual science; Pharmacology and drug therapy in eye diseases; Basic Sciences; Primary and Secondary eye care; Patient Safety and Quality of Care Improvements. This journal is indexed on Submit your manuscript here: http://www.dovepress.com/clinical-ophthalmology-journal

\section{Dovepress}

PubMed Central and CAS, and is the official journal of The Society of Clinical Ophthalmology (SCO). The manuscript management system is completely online and includes a very quick and fair peer-review system, which is all easy to use. Visit http://www.dovepress.com/ testimonials.php to read real quotes from published authors. 